\title{
Integrated Anti-Icing Property of Super-Repellency and Electrothermogenesis Exhibited by PEDOT:PSS/Cyanoacrylate Composite Nanoparticles
}

Takeshi Matsubayashi,$^{\dagger}$ Mizuki Tenjimbayashi ${ }^{\dagger}$ Kengo Manabe,$^{\dagger}$ Masatsugu Komine,$^{\dagger}$ Walter Navarrini $i^{\sharp}$ and Seimei Shiratori ${ }^{*}{ }^{\dagger}$

${ }^{\dagger}$ Center for Material Design Science, School of Integrated Design Engineering, Keio University, 3-14-1 Hiyoshi, Kohoku-ku, Yokohama, Kanagawa 223-8522 Japan

${ }^{\ddagger}$ Dipartimento di Chimica, Materiali e Ingegneria Chimica, Politecnico di Milano, via Mancinelli 7, I-20131, Milano, Italy

*shiratori@appi.keio.ac.jp 
Movie S1| Movie of water droplet impacting on the bare PET substrate taken by high speed camera $(W e=38.2)$.

Movie S2 $\mid$ Movie of water droplet impacting on the $\mathrm{P}_{30}$ surface taken by high speed camera $(W e=38.2)$.

Movie S3| Movie of water droplet impacting on the $\mathrm{P}_{30}$ surface taken by high speed camera $(W e=80.3)$.

Movie S4| Defrosting movie of the $\mathrm{P}_{30}$ film performed before and after applying the DC voltage of $18 \mathrm{~V}$.

Movie S5 $\mid$ A movie illustrating water-repellent properties of the bent $\mathrm{P}_{30}$ film with bent. Movie S6 $\mid$ A movie of supercooled mist impacting on the $\mathrm{P}_{30}$ surface captured by high speed camera.

Movie S7 $\mid$ A movie of acid water ( $\mathrm{pH}=5.6$, assuming acid rain) impacting on the $\mathrm{P}_{30}$ surface captured by high speed camera. 


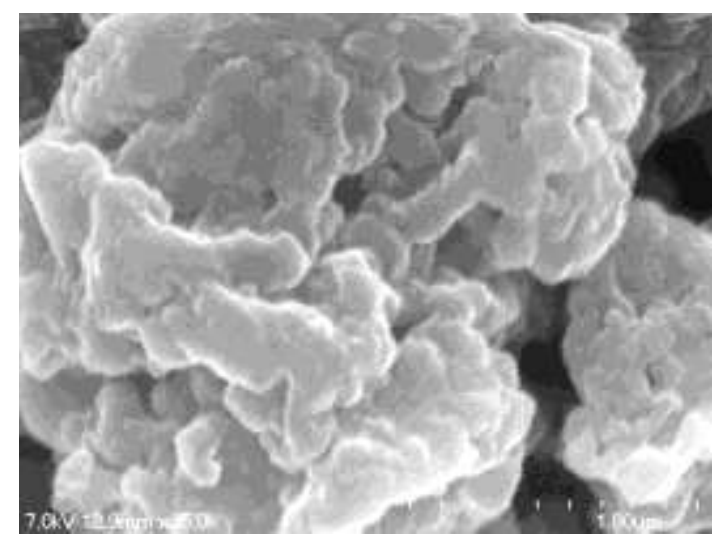

Figure S1. SEM image of $\mathrm{P}_{30}$ surfaces in high magnification. 


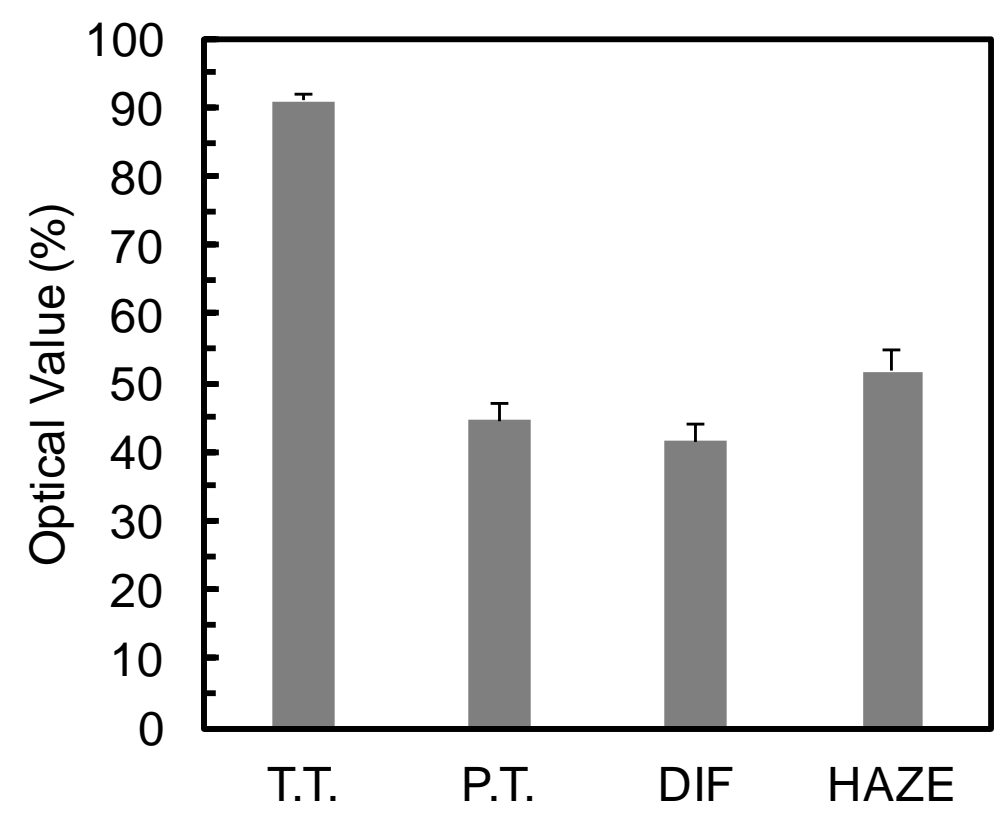

Figure S2. Optical values of $\mathrm{P}_{30}$ film. Parallel transmittance (P.T.) means the degree that light passes through a sample within a small range of angle $\left(<15^{\circ}\right)$ and diffusion (DIF) is a scattering of light when it passes through the sample. Total transmittance (T.T.) is the sum of P.T. and DIF. HAZE is calculated as (DIF/T.T.) $\times 100$. 


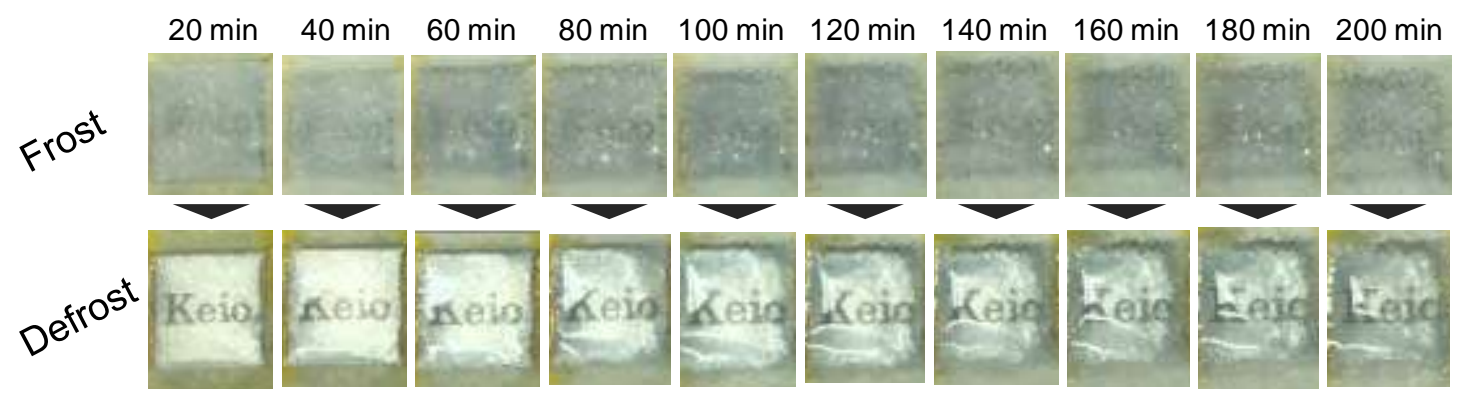

Figure S3. 10 cycles of frosting/defrosting performance of conductive superhydrophobic surface. During the periodic defrosting testing, a heater was placed on a Peltier cooler with a temperature of $-20{ }^{\circ} \mathrm{C}$, which was located inside a chamber with internal temperature of $10{ }^{\circ} \mathrm{C}$ and relative humidity of $80 \%$ (to promote frost formation). Subsequently, a DC voltage of $18 \mathrm{~V}$ was applied for $90 \mathrm{~s}$ to the heater contacts to defrost its surface under the same conditions. Frost was melt even after $10^{\text {th }}$ cycle and the background characters can be clearly seen through the transparent film. 
(a)

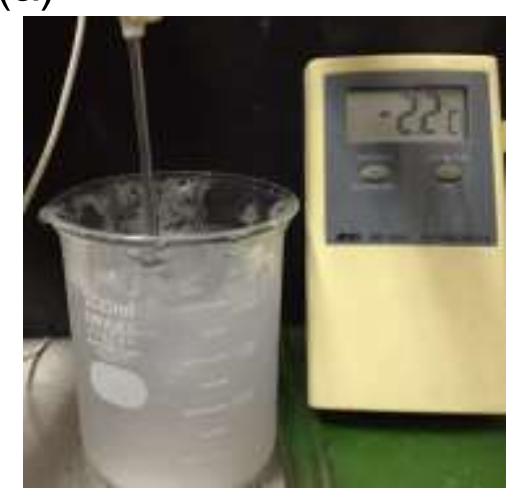

(b)

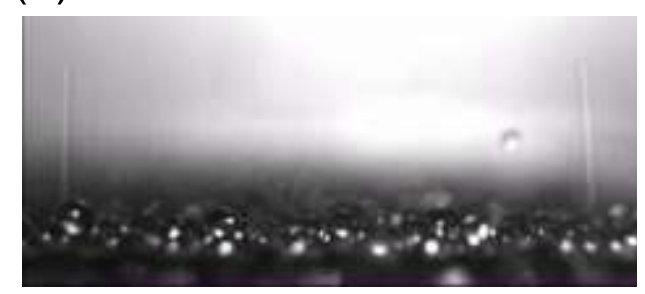

(c)

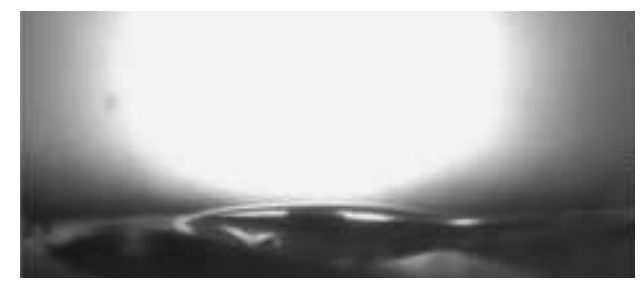

Figure S4. (a) Setup for preparing supercooled water, which was cooled by the mixture

of ice and salty water. Pictures after spraying misty supercooled water onto (b) $\mathrm{P}_{30}$ surface and (c) bare PET substrate. Misty supercooled water $(0.15 \mathrm{~mL})$ was sprayed from $15 \mathrm{~cm}$ above onto $\mathrm{P}_{30}$ surface, on which the milli-sized droplet was deposited in spherical shape with reduced contact area, whereas bare PET surface was fully wetted. 
(a)

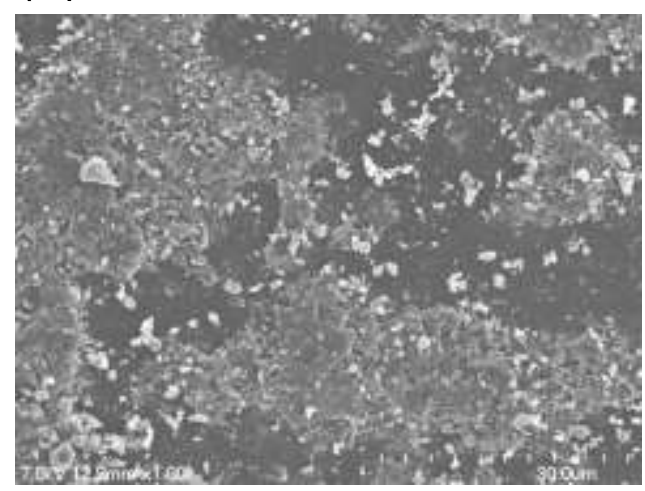

(b)

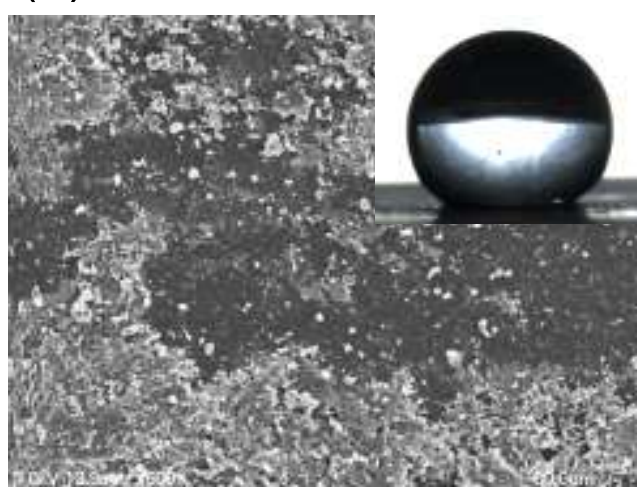

Figure S5. SEM images of conductive superhydrophobic surface after (a) 100 and (b)

140 times of abrasion. As shown in the inset image of (b), the surface loses their superhydrophobicity (contact angle of $149^{\circ}$ ) after 140 times of abrasion. 


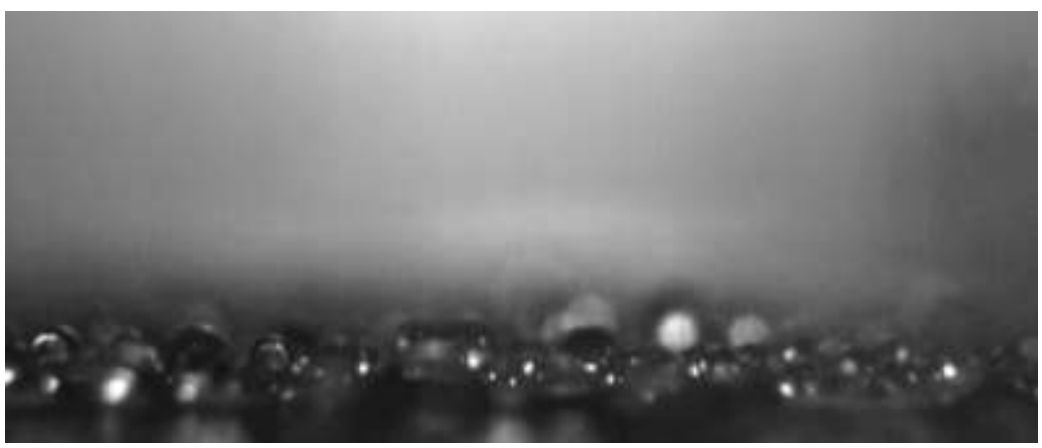

Figure S6. Picture of $\mathrm{P}_{30}$ surface after sprayed acidic mist. Misty acid water $(0.15 \mathrm{~mL}$, $\mathrm{pH}=5.6$ ) was sprayed from $15 \mathrm{~cm}$ above onto $\mathrm{P}_{30}$ surface, assuming acid rain. Milli-sized droplet was deposited in spherical shape with reduced contact area and the droplet was easily removed by small force, which indicate no influence of acid rain. 
We fabricated two layer coating of conductive layer and superhydrophobic layer to compare with our conductive superhydrophobic films. Firstly we tried fabricating PEDOT:PSS layer for conductive layer. PEDOT:PSS water-based solution (Orgacon S305) was spin coated on the $\mathrm{UV} / \mathrm{O}_{3}$ treated PET substrate at $1000 \mathrm{rpm}$ for $10 \mathrm{~s}$. However, PEDOT:PSS layer was broken and peeled off from substrate after air-blowed by sprayer with $0.7 \mathrm{MPa}$ from $40 \mathrm{~cm}$ (Figure S7).

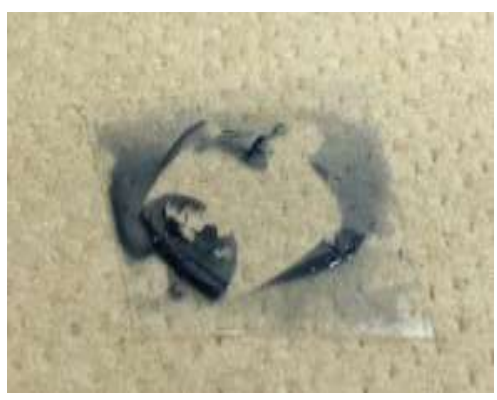

Figure S7. Spin coated PEDOT:PSS layer after air-blowed by sprayer.

Therefore, a tin-doped indium oxide (ITO) was then selected as conductive layer because of its wide use as a heating element. Hydrophobic $\mathrm{SiO}_{2}$ nanoparticles (Aerosil RX 200, diameter $\sim 12 \mathrm{~nm}$, Evonik Industries, Germany) was sprayed onto ITO substrate by dispersing 4 wt.\% of $\mathrm{SiO}_{2}$ in Ethanol. 
Fabricated two layer coating was subjected to $2 \mathrm{kPa}$ abrasion pressure, same as the abrasion test in main manuscript. Figure S8 shows the surface lost its superhydrophobicity after 5 cycles of abrasion (blue line). Our conductive superhydrophobic films have superior abrasion resistance (maintain superhydrophobicity after 100 abrasion cycles) because of their inter-connected structures (Fig. 1).

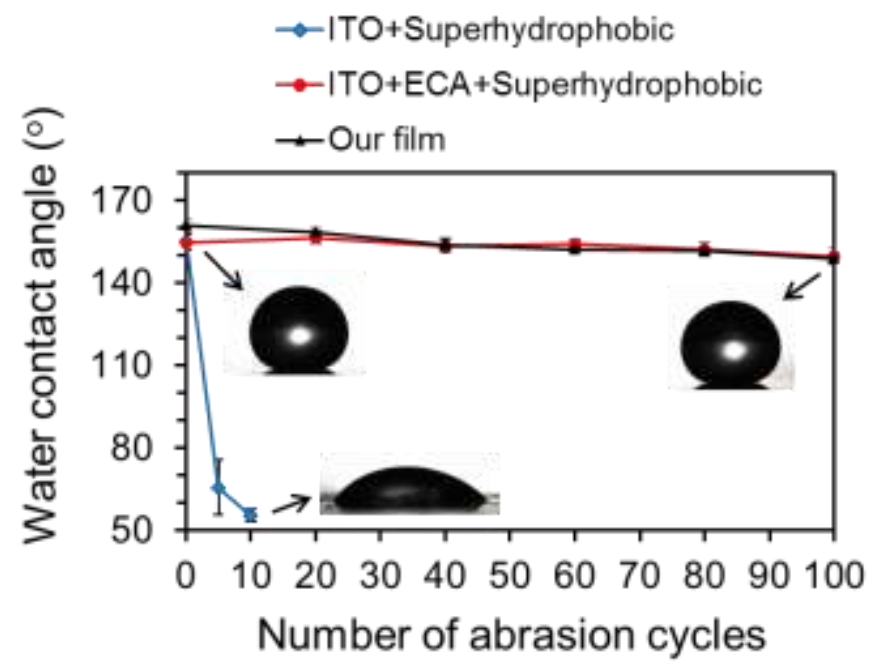

Figure S8. Water contact angle of two layer conductive superhydrophobic coating (ITO + hydrophobic $\mathrm{SiO}_{2}$ and ITO + ECA resin + hydrophobic $\mathrm{SiO}_{2}$ ) under repeated abrasion cycles with sandpaper.

To improve the durability of superhydrophobic coating, we mixed ethyl cyanoacrylate (ECA) with hydrophobic $\mathrm{SiO}_{2}$ as adhesive resin. ${ }^{48} 4$ wt.\% of $\mathrm{SiO}_{2}$ and 
ECA were dispersed in acetone and sprayed to ITO substrate. With ECA, fabricated surface maintained superhydrophobicity after 100 cycles of abrasion under $2 \mathrm{kPa}$ (Fig. S2). However, optical transparency was deteriorated as shown in Figure S9. In addition, two layer coating did not show electric conductivity with conductive layer coated by superhydrophobic layer.

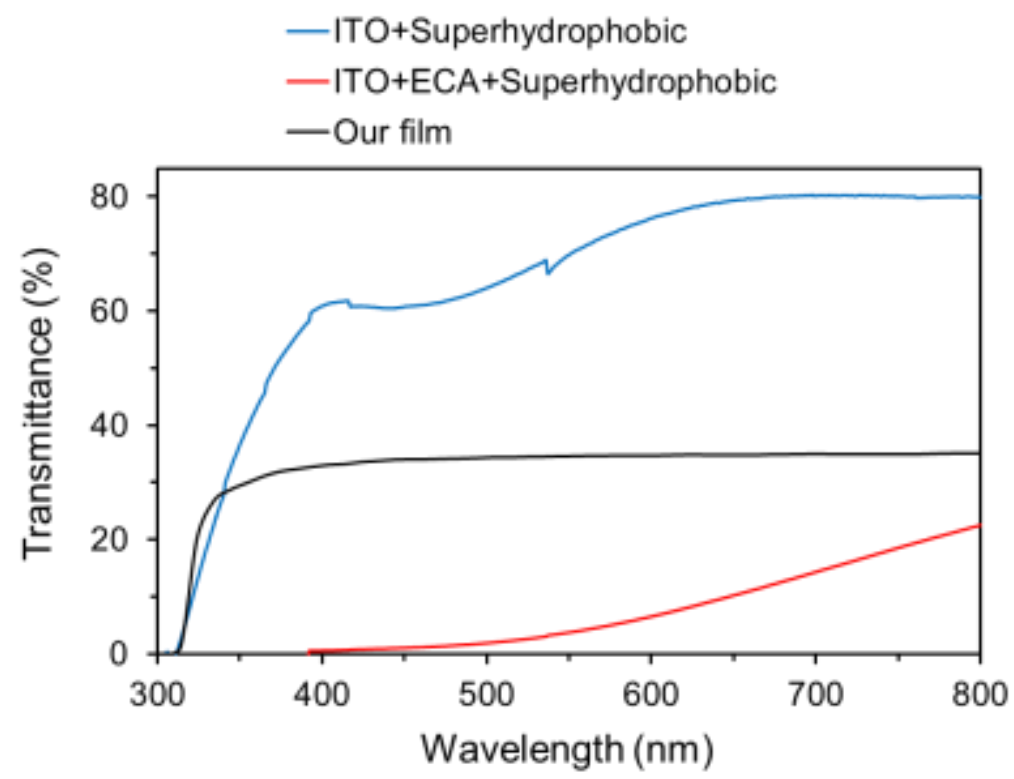

Figure S9. Optical transparency of ITO substrate and superhydrophobic coated ITO.

From a view point of a heater, our film demonstrated higher energy efficiency $\left(260.8{ }^{\circ} \mathrm{C}\right.$ $\left.\mathrm{cm}^{2} / \mathrm{W}\right)$ than ITO heater $\left(88{ }^{\circ} \mathrm{C} \mathrm{cm}^{2} / \mathrm{W}\right) .^{49}$ Furthermore, ITO is prohibitive in large area or flexible application because of indium scarcity, crack formation under bending. 
Therefore, our conductive superhydrophobic film has advantage against two layer coatings in terms of mechanical durability and heating energy consumption, and one-step spray coating of conductive polymer nanoparticles is considered to be suitable for large area application.

(48) Sasaki, K.; Tenjimbayashi, M.; Manabe, K.; Shiratori, S. Asymmetric Superhydrophobic/Superhydrophilic Cotton Fabrics Designed by Spraying Polymer and Nanoparticles. ACS Appl. Mater. Interfaces 2016, 8, 651-659.

(49) Ji, S.; He, W.; Wang, K.; Ran, Y.; Ye, C. Thermal Response of Transparent Silver Nanowire/PEDOT:PSS Film Heaters. Small 2014, 10, 4951-4960. 